\title{
Loss Minimization in Distribution Network using Wind Power Plant Reactive Power Support
}

\author{
Aeishwarya Baviskar, Student Member, Kaushik Das, Senior Member, IEEE, Panagiotis Menegatos, \\ Anca Daniela Hansen
}

\begin{abstract}
The increased penetration of wind power plants (WPPs) in distribution networks challenges the distribution system operators (DSOs) to improve and optimize networks' operation. A higher amount of local power production translates to more losses in the network. This paper proposes a deterministic optimization methodology to minimize the losses in distribution networks with WPPs, by exploiting WPPs' capability to control reactive power in coordination with the on-load tap changers from the MV/HV transformer, avoiding the need for network reinforcements. The principal objective is to optimize the reactive power flow in the network. Measurements from a real distribution network with a large share of controllable WPPs under varying wind and load conditions are used for the study. The benefits and the challenges of the optimization methodology are assessed and discussed with respect to active power losses, voltage profile and reactive power. The results show that with reactive power support from WPPs, network losses are reduced by $4.2 \%$. Higher loss reductions (up to $19 \%$ ) can be achieved through a coordinated action between the WPPs and TSO. Furthermore, it is shown that the distribution network can act as an asset to the transmission network for reactive power support, via actively controlling WPP's reactive power.
\end{abstract}

Index Terms-loss minimization, wind power plants, reactive power set-points, OLTC settings, grid codes, capability curve

\section{INTRODUCTION}

$\mathbf{I}$ ncreasing concern for climate change has led to increasing share of renewable energy sources (RES) in power systems around the world. RES can either be connected as utility scale power plants in bulk-power transmission systems or as distributed generations connected to distribution systems. The total distributed wind generation capacity installed in Denmark alone, in 2020 was around $3.1 \mathrm{GW}$, which is $\approx 50 \%$ of Denmark's total installed wind generation capacity [1]. In $2019,50 \%$ of the total electricity consumed in Denmark was generated by renewable energy sources of which $47 \%$ came from wind power plants (WPPs) [2]. The European grid codes require WPPs to provide support to the grid in terms of active power control, reactive power supply, maintaining power quality and voltage profiles, fault-ride through capability and protection [3]. At the distribution level, integration of WPPs offer several such benefits, like reducing congestion and power losses in the transmission lines, active support in terms of voltage stability, improved load shedding, etc [4]. With improved technology and reducing cost of installations,

Aeishwarya Baviskar, Kaushik Das and Anca Daniela Hansen are with the De partment of Wind Energy, Technical University of Denmark, Frederiksborgvej 399, 4000 Roskilde, Denmark (e-mail: kdas@dtu.dk). Panagiotis Menegatos is with Siemens Gamesa Renewable Energy, Denmark and was in DTU Wind Energy when he contributed to this work.
WPPs are a prevalent choice among different RES options to support the network, especially when the benefits for the distribution system operators (DSOs) are not only technical but also economical [5]. However, nowadays, due to increase in the share of WPPs and other RES in distribution grids, the DSOs face additional challenges concerning under- and overvoltages, higher active power losses, transformer and cable overloading, etc. [6]-[10].

One of the main challenges behind integrating a large share of RES such as WPPs in distribution grids is the increase in active power losses. Note that the power generated due to WPPs is weather-dependent and non-dispatchable in nature. When local active power injection is introduced in the network, active power losses decrease up to a certain amount of installed capacity. Beyond this break point, reverse power flow in the network increases resulting in an increase in active power losses, as demonstrated in [6]. Injection of active power at the end of the feeders increases the voltage levels at the receiving end of the feeders which disrupts the traditional design of a radial feeder [7]. Especially during high generation and low load scenarios, the increase in voltage could be detrimental. Distribution system losses and the voltage profiles have become a significant concern for the distribution grid [8], [11]. Thus, minimizing the distribution system losses in presence of wind power has been a prevalent research topic over the years [4], [12]-[19]. Optimal allocation and placement of WPPs in the distribution grid is one of the many strategies explored for effective integration of WPPs while facilitating low network losses and steady voltage profiles [6], [20]. Allocation studies for placing WPPs or network assets like capacitors, voltage regulators, on-load tap changers (OLTCs) etc. are however less relevant for a network with pre-existing high share of WPPs, and might not be always reasonable to limit the producers to connect at the nearest connection point. It thus becomes imperative to utilize the capabilities of already existing WPPs and network assets such as OLTCs, voltage regulators, switching capacitors, etc. to support the network to minimize losses, maintain voltage profiles, and provide reactive power support to the distribution grid. Using WPPs along with network assets to reduce network losses provides economic benefit for the DSOs and saves costs on further network reinforcements.

The concept of using WPP or distributed generation capabilities has been tested in many works. In an earlier work, authors used reactive power from wind farms to maintain system voltage in steady state and during grid disturbance using a particle swarm based optimization method (PSO) [12]. Similarly, a WPP connected to a German sub-transmission 
system was used in [21] to provide the reactive power needs of the sub-transmission and the transmission network, also based on PSO. A co-ordinated control approach between installed capacitors and distributed energy resources (DERs) is deployed in [22], to increase energy efficiency, whereas; [13] implements optimal power flow to determine optimal reactive power in a network with WPPs to minimize losses. An example of deploying DERs' capability to maintain voltage profile using a deterministic optimization can be found in [23]. However, authors in [23] maintain a voltage profile at the point of connection of the DER and resort to active power curtailment if reactive power limits are reached. In [24], the objective is to reduce the OLTC operations by dispatching reactive power from inverter based RES. However, the results are supported based on simulations over a 24 hour timeseries on a distribution grid with $80 \%$ penetration of RES (photovoltaics PVs and WPPs). The complementary nature of PV and WPPs is used in [16] for power loss minimization. Authors in [25] implement a droop control for renewable generators in the distribution grid for voltage support. The fuzzy characteristics of distributed generation sources are exploited in [19] to optimize distribution grids' reactive power and to minimize expectation of network losses and voltage deviation. A more recent study shows capability of distributed generation sources, such as WPPs and PVs, to provide voltage ancillary services [26]. Authors in [27] again provide an example of using reactive power control on DERs for handling voltage violations in the network. However, the primary response to voltage violations in [27] comes from OLTC settings and DER capabilities are deployed as a fast corrective control. Most of these literature's focus on utilizing reactive power from WPPs or DER has voltage regulation as the primary objective and loss minimization as a secondary objective. In addition, reactive power from WPPs is more often deployed as a secondary measure while primary measure of control remains with network assets.

However, the effects of using the reactive power capabilities of WPPs in a distribution grid, without active power curtailment, on distribution system losses are relatively unexplored for longer horizon measurement data. Moreover, the impacts of local generation/absorption of reactive power by WPPs on the DSO/TSO reactive power exchange have not been yet investigated in details in the existing literature.

The main objective of this paper is to develop and present an optimization methodology to minimize the losses in distribution networks with large share of wind power, exploiting WPPs capability to control reactive power in coordination with the on-load tap changers from the medium/high voltage $(\mathrm{MV} / \mathrm{HV})$ transformer. Key contributions of this paper are listed as follows:

1) Develop novel methodology to reduce network losses in a distribution network with a large share of WPPs, by exploiting WPPs' control capabilities to absorb or consume reactive power.

2) Assess the value of utilizing the entire reactive power capability of a WPP beyond the grid-code requirements to reduce active power losses in the network.
3) Depict the value of the control of MV/HV OLTC transformer for active power loss reduction.

4) Quantify maximum loss reduction by employing WPPs' control capabilities along with and tap-changer settings.

An in-depth analysis of reactive power exchange between distribution and transmission network is presented to explore co-ordination opportunities between TSO and DSO. This investigation is carried out using the measurement data of a realtime distribution network, described in [4]. Wherein a detailed analysis of the active and reactive power flows in a real distribution network based on actual measurements, as well as of the co-relation between the consumption, wind power production and network losses is described. The preliminary analysis results presented in [4], shows that the network losses are proportional to the wind power production. This result is used as a base in the present research investigation. The paper is structured as follows. Section II describes the distribution network model. The said methodology is presented in Section III, while in Section IV, sheds light on the implementation details. Results from the simulation are presented and described in Section V. Finally, conclusive remarks are presented in Section VI, where the track for future work is also proposed.

\section{Distribution Network Model}

A distribution network model, based on a real Danish medium voltage (i.e. $60 \mathrm{kV}$ ) distribution network, is briefly described in the following. Further details can be found in [4], [28]. Fig.1 illustrates the layout of this network, which is connected to the high voltage (HV) transmission network through one step-up $60 / 150 \mathrm{kV}$ transformer, and to the low voltage (LV) $10 \mathrm{kV}$ buses through several step-down $60 / 10 \mathrm{kV}$ transformers. Three controllable WPPs are connected to this distribution network, i.e. the $W P P_{1}$, rated $12 \mathrm{MW}$, and $W P P_{2}$ and $W P P_{3}$ rated $15 \mathrm{MW}$ each.The salient features of this network are tabulated as follows: Almost one year of SCADA

TABLE I: The $60 \mathrm{kV}$ distribution network

\begin{tabular}{|l|c|r|}
\hline Network Element & $\#$ & Voltage Level \\
\hline Buses & 25 & $60 \mathrm{kV}$ \\
\hline TSO/DSO substation & 1 & $150 \mathrm{kV} / 60 \mathrm{kV}$ \\
\hline Consumer substation & 6 & $60 \mathrm{kV} / 10 \mathrm{kV}$ \\
\hline Substations with uncontrollable generation & 13 & $60 \mathrm{kV} / 10 \mathrm{kV}$ \\
\hline Substations with controllable generation & 3 & $60 \mathrm{kV} / 10 \mathrm{kV}$ \\
\hline Transformers & 1 & $150 \mathrm{kV} / 60 \mathrm{kV}$ \\
& 22 & $60 \mathrm{kV} / 10 \mathrm{kV}$ \\
\hline Type IV controllable WPPs & 3 & $60 \mathrm{kV}$ \\
\hline Loads (Aggregated) & 22 & $10 \mathrm{kV}$ \\
\hline
\end{tabular}

measurements with one-hour resolution is available for the present investigation.

\section{OPTIMIZATION METHODOLOGY}

The optimization problem defined in this research is highly nonlinear, non-convex, constrained and involves discrete and continuous variables. It can be formulated as shown below, with $f(x, u(x))$ denoting the objective function, while 


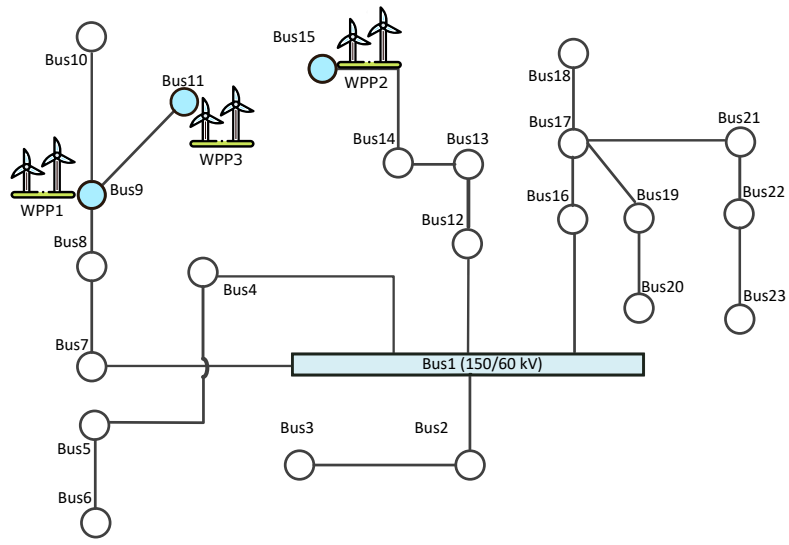

Fig. 1: Layout for the $60 \mathrm{kV}$ distribution network

$h(x, u(x))$ and $g(x, u(x))$ denoting the equality and inequality constraints respectively:

$$
\begin{aligned}
\min & f(x, u(x)) \\
\text { s.t. } & h(x, u(x))=0 \\
& g(x, u(x)) \leq 0 \\
& x^{l} \leq x \leq x^{u}
\end{aligned}
$$

where $x$ represents the control variable, and $u(x)$ is a function of the control variable, while $x^{l}$ and $x^{u}$ are lower and upper limits for the control variables, respectively.

\section{A. Objective function}

The objective of this optimization is to reduce the total active power losses $P_{L}$ in the network, given by,

$$
P_{L}=\sum_{(i, j) \in \mathcal{E}_{\mathcal{B}}} G_{i j}\left(V_{i}^{2}+V_{j}^{2}-2 V_{i} V_{j} \cos \left(\theta_{i j}\right)\right)
$$

where $i, j$ are the indices of the set of branch edges $\left(\mathcal{E}_{\mathcal{B}}\right)$ of the system. $G_{i j}$ is the series conductance of the line connecting sending end $i$ and receiving end $j . V_{i}, V_{j}$ are the voltages and $\theta_{i j}$ is the difference in the phase angles of the voltages at the $i$ and $j$ ends of the line.

\section{B. Control variables}

The control variables used in this work are:

- WPP reactive power set-points $Q_{W P P_{j}}$ for all three WPPs (i.e. $j=1,2,3$ ). Notice that $W P P_{j}$ is connected to bus $i \in \mathcal{N}_{\mathcal{W P P}}$;

$\mathcal{N}_{\mathcal{W P P}}$ is the set of buses with controllable WPPs

$\mathcal{N}_{\mathcal{W P P}} \subseteq \mathcal{N}_{\mathcal{B}}$.

$\mathcal{N}_{\mathcal{B}}$ is the set of all buses in the network

- Tap-settings $T_{M V}$ for $60 / 150 \mathrm{kV}$ step-up transformer

WPPs can operate in different control modes, namely they are capable of providing or absorbing reactive power by either voltage, reactive power or power factor control during steadystate operation [29]. As the reactive power capability of WPPs is dependent on both active power production $P_{W P P}$ and voltage $V_{W P P}$, an optimization using reactive power $Q_{W P P_{j}}$ as control variable $(x)$, with lower $x^{l}\left(P_{W P P}, V_{W P P}\right)$ and upper $x^{u}\left(P_{W P P}, V_{W P P}\right)$ variable limits, such as

$$
x^{l}\left(P_{W P P}, V_{W P P}\right) \leq x \leq x^{u}\left(P_{W P P}, V_{W P P}\right)
$$

is difficult to implement.

The control of the WPPs reactive power in this investigation is therefore performed indirectly by altering the voltage at the point of connection (PoC) and changing thus the WPPs reactive power output. In addition, tap-settings of the stepup transformer (i.e. $60 / 150 \mathrm{kV}$ ) can also be considered as a control variable to improve voltage profile in the network. The voltage level of the OLTC transformer is controlled on the low $60 \mathrm{kV}$ side, directly via tap-settings. The control vector of the optimization problem hereby consists only of voltage control variables, as follows:

$$
x=\left[V_{W P P_{1}}, V_{W P P_{2}}, V_{W P P_{3}}, V_{T_{M V}}\right]^{T}
$$

where $V_{W P P_{j}}$ is the voltage at the PoC for $W P P_{j}$ with $j=1,2,3$ and $V_{T_{M V}}$ is the voltage for the $60 / 150 \mathrm{kV}$ transformer. It is important to notice that all the control variables are bounded by upper $\left(x^{u}=1.1\right.$ p.u. $)$ and lower $\left(x^{l}=0.9\right.$ p.u. $)$ voltage levels according to the grid code requirements [30].

\section{Constraints}

1) Equality constraints: The equality constraints of the optimization problem are the active and reactive power balance equations for each bus $i$ at each time-step $t$, accounted directly inside the load-flow algorithm:

$$
\begin{array}{cc}
P_{D_{i, t}}-P_{G_{i, t}}+P_{I_{i, t}}=0 & \forall i \in \mathcal{N}_{\mathcal{B}} \\
Q_{D_{i, t}}-Q_{G_{i, t}}+Q_{I_{i, t}}=0 & \forall i \in \mathcal{N}_{\mathcal{B}}
\end{array}
$$

where $P_{D_{i, t}}$ and $Q_{D_{i, t}}$ represent the active and reactive power demand of the $i^{t h}$ bus at time $t$, respectively. $P_{G_{i, t}}$ and $Q_{G_{i, t}}$ are the generated active and reactive power at the $i^{t h}$ bus at time $t$,respectively, while $P_{I_{i, t}}$ and $P_{I_{i, t}}$ denote the active and reactive power injections into the $i^{\text {th }}$ bus at time $t$, respectively.

2) Inequality Constraints: Four types of inequality constraints are considered in the optimization problem, e.g. WPP generation constraints, transformer constraints, security constraints and MVAr transfer constraints.

WPP generation constraints - these are inequality constraints on the voltage at PoC for WPPs, and their reactive power capability.

$$
\begin{aligned}
V_{W P P_{j}}^{l} \leq V_{W P P_{j, t}} \leq V_{W P P_{j}}^{u} \quad \forall j \\
Q_{W P P_{j}}^{l} \leq Q_{W P P_{j, t}} \leq Q_{W P P_{j}}^{u} \quad \forall j
\end{aligned}
$$

where $V_{W P P_{j, t}}$ and $Q_{W P P_{j, t}}$ is the voltage and reactive power output of $W P P_{j}$ at time $t$, respectively. $V_{W P P_{j}}^{l}$ and $V_{W P P_{j}}^{u}$ are the lower and upper voltage limits, and similarly $Q_{W P P_{j}}^{l}$ and $Q_{W P P_{j}}^{u}$ are the lower and upper reactive power limits. The WPP upper and lower voltage limits are set according by the grid codes [30] as specified in section III-B. As depicted in Fig. 2, the grid code requirements $(G C R)$ for WPPs provide a feasible area for WPPs reactive power output $Q_{W P P}$ 
in relation to their active power output and the voltage at $\mathrm{PoC}$. In comparison with the reactive power capability curves $(C C)$ (Fig. 4) however; GCR offer a restrictive operating zone.

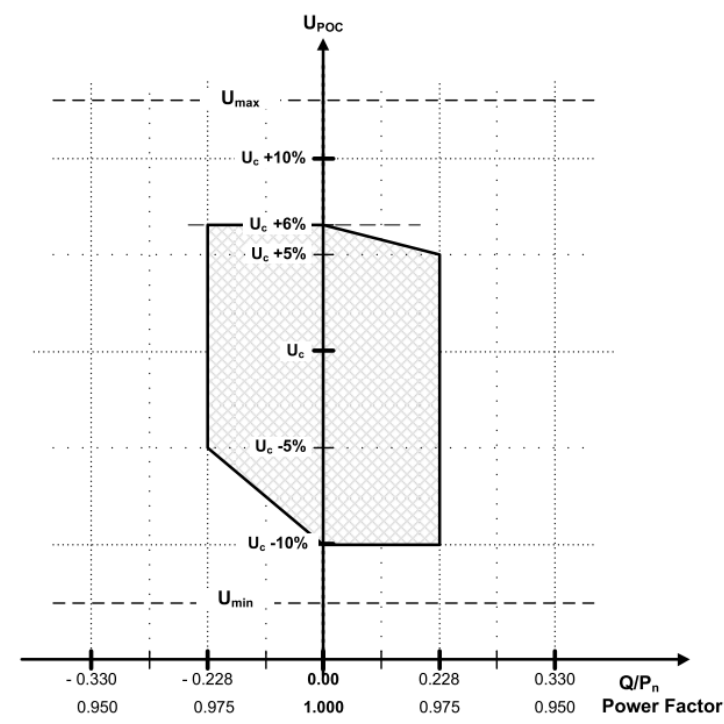

Fig. 2: Grid code requirements $(G C R)$ for delivery of reactive power in relation to voltage in $\mathrm{PoC}[30]$.

The present research assumes that the wind turbines installed in the $60 \mathrm{kV}$ network are Type IV wind turbines connected by a back-to-back converter to the grid. Voltage and current limitations of the grid side converter predominantly limit the reactive power from wind turbine [31]. Fig. 3 gives a simplification of the WT system by depicting only the grid side converter by an equivalent impedance $Z_{W T}$.

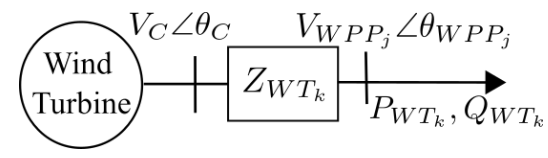

Fig. 3: Simplified representation of wind turbine's grid side converter connected to WPP collection system through WT transformer [31]

$V_{c}$ and $\theta_{c}$ in Fig. 3 represent the converter voltage magnitude and angle, $V_{W P P_{j}}$ and $\theta_{W P P_{j}}$ represent voltage magnitude and angle at point of connection to WPP collection system, namely MV side of wind turbine's transformer, $Z_{W T_{k}}$ denotes an equivalent impedance from the grid side converter up to the high voltage side of the wind turbine transformer, and $P_{W T_{k}}$ and $Q_{W T_{k}}$ are the active and reactive power output from the wind turbine $k$ in the $W P P_{j}$.

$$
Z_{W T_{k}}=\sqrt{R_{W T_{k}}^{2}+X_{W T_{k}}^{2}}
$$

where, $R_{W T_{k}}$ and $X_{W T_{k}}$ represent equivalent resistance and impedance of the grid side converter. Assuming $\mathcal{K}_{\mathcal{W T}}$ is the set of all WTs in $W P P_{j}$ system,

$$
\begin{aligned}
P_{W P P_{j}}=\sum P_{W T_{k}} & \forall k \in \mathcal{K}_{\mathcal{W T}} \\
Q_{W P P_{j}}=\sum Q_{W T_{k}} & \forall k \in \mathcal{K}_{\mathcal{W T}}
\end{aligned}
$$

As demonstrated in [31], the wind turbine' reactive power limited by converter voltage $Q_{W T_{k}, V}$ can be written as follows,

$$
\begin{aligned}
Q_{W T_{k}, V}= & \sqrt{\left(\frac{V_{W P P_{j}} V_{C}}{Z_{W T_{k}}}\right)^{2}-\left(P_{W T_{k}}+\frac{V_{W P P_{j}}^{2} R_{W T_{k}}}{Z_{W T_{k}}}\right)^{2}} \\
& -\frac{V_{W P P_{j}}^{2} X_{W T_{k}}}{Z_{W T_{k}}}
\end{aligned}
$$

By replacing $V_{C}$ in the Eq. (10) with the maximum and minimum allowable converter voltage $V_{C \max }$ and $V_{C \min }$, the maximum injection and absorption of reactive power limited by converter voltage can be calculated, respectively. Similarly, as written in [31], the reactive power limited by grid side converter current $Q_{W T_{j}, I}$ can be calculated as follows:

$$
Q_{W T_{k}, I}= \pm \sqrt{\left(V_{W P P_{j}} I_{C \max }\right)^{2}-P_{W T_{k}}^{2}}
$$

where $I_{C \max }$ is the maximum converter current limit. The positive and negative roots in Eq.(11) indicate the maximum reactive power injection and absorption limited by converter current. Authors in [31] provide a comprehensive derivation of the above equations.

Fig.4 shows reactive power capability curves (CC) in relation to the active power output of the WPPs at different voltage levels. These non-linear reactive power capability curves are derived based on Eq. (10) and Eq. (11). The reactive power output $\left(Q_{W P P_{j}}\right)$ of $W P P_{j}$ is a function of the active power and voltage on the high voltage side $V_{H V}$ of the WT transformer. Solid lines in Fig. 4 denote the upper limit of the reactive power injection capability, whereas, dotted lines denote the upper limit of the reactive power absorption capability of the WPPs at a particular voltage level. As WPPs operate in voltage control mode, the upper and lower bounds of the reactive power $Q_{W P P_{j}}$ can be easily calculated for each time-stamp. Thereby, as the control variable is adjusted towards the optimal solution, the upper and lower bounds for $Q_{W P P_{j}}$ are adjusted considering its current $P_{W P P_{j}}, V_{W P P_{j}}$ level. Notice from Fig. 4 that, as the voltage level on the high voltage side increases, for the same amount of active power production $P_{W P P_{j}}$, the reactive power capability of WPPs decreases. Based on WPP's capability curves, it is thus worth noting that by raising the voltage level to decrease current and thus the active power losses, will limit the WPP's reactive power capability.

Transformer constraints - these are minimum and maximum constraints on the transformer tap-setting as follows,

$$
T_{M V}^{\min } \leq T_{M V} \leq T_{M V}^{\max }
$$

It should be mentioned that, in this investigation, the transformer tap-setting is considered as a continuous variable and rounded to the closest integer settings after the load flow converges. Since, tap-settings are modified indirectly via the control variable $V_{T_{M V}}$ (as given by Eq. (3)), the tap-setting constraints are also incorporated indirectly via an OLTC adjustment module. 


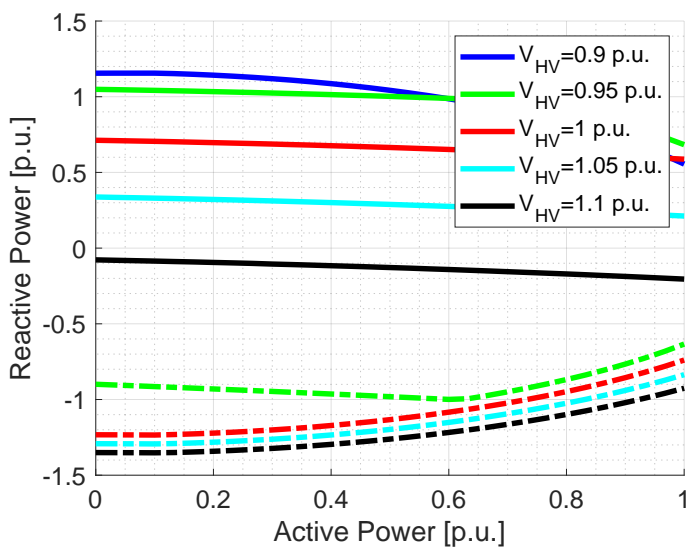

Fig. 4: Reactive power capability curve of a typical Type IV WT for different voltages levels on the HV side [32]

Security constraints - these are inequality constraints, which define the lower and upper voltage limits for all the buses, and the maximum loading for each bus.

$$
\begin{gathered}
V_{i}^{l} \leq V_{i} \leq V_{i}^{u} \quad \forall i \in \mathcal{N}_{\mathcal{B}} \\
S_{i} \leq S_{i}^{\max } \quad \forall i \in \mathcal{E}_{\mathcal{B}}
\end{gathered}
$$

where $V_{i}$ is the voltage at the $i^{\text {th }}$ bus and $S_{i}$ is the power flow in the distribution line.

MVAr transfer constraint - this is the constraint which can be imposed by a TSO on the MVAr flow between distribution and transmission networks. For example, in Denmark, the reactive power transfer range between the distribution and the transmission network should be limited to $48 \%$ of the MVA capability of the MV/HV transformer [30]. In this present investigation, as the MVA capability of the MV/HV transformer is $160 \mathrm{MVA}$, the reactive power transfer limit from the transmission network to the distribution network is limited to $\pm 76.8 \mathrm{MVAr}$.

\section{IMPLEMENTATION}

The proposed optimization methodology to minimize active power losses is an iterative procedure implemented in Python. PYPOWER package is used to implement the network model. The algorithm receives hourly SCADA measurements for active and reactive power, voltage, and current. First, the measurements are filtered, any missing data is filled in using a state estimator, while bad data is eliminated. The network model is updated with the current measurements every hour. Using the latest measurements, the optimization routine works towards reducing the active power loss for each particular hour.

As output, the optimization provides voltage setting for the WPPs and $60 / 150 \mathrm{kV}$ transformer. The OLTC adjustment algorithm is then called to determine the tap-setting for the $60 / 150 \mathrm{kV}$ transformer as per the control variable $\left(V_{T_{M V}}\right)$ from the optimization routine. The tap-setting for the transformer is calculated based on the first-order sensitivity factor for change in the regulated nodal voltages and line's active power with respect to change in tap-settings of the transformer.
To this end, a modified Jacobian matrix is calculated. Details regarding the OLTC adjustment module are elaborated in [33].

A Newton-Raphson based load-flow calculates the steadystate variables such as voltage magnitudes and angles for all buses, active and reactive power flows in each line, and active power loss for each iteration step for every hour. It is important to highlight again, that, the present routine enables the control of bus voltages instead of the tap-settings. and this significantly simplifies the control vector to only consists of voltages.

\section{REsults}

In this section, the proposed optimization method to minimize the losses in distribution networks with large share of WPPs, by utilizing wind turbines control capabilities is assessed and discussed with respect to active power losses, voltage profile and reactive power for a set of simulated cases.

\section{A. Study cases}

Different study cases are carried out on the real Danish distribution network, depicted in Fig.1. Data from one year SCADA measurements with resolution of an hour for load and generation profiles are available for the period between $8^{\text {th }}$ Dec-2014 and $31^{\text {st }}$ Oct-2015. Table II provides an overview of all the simulated cases, described in the following.

Base Case - used as reference, is defined as the distribution network case when WPPs reactive power control capabilities are not used, namely when WPPs do not contribute with reactive power to the network, being operating at a predefined reactive power set-point, which is typically 0 MVA.

WPP $(\boldsymbol{G C R})$ or $\boldsymbol{W P P}(\boldsymbol{C C})$ cases - two cases where the constraints, on WPPs' flexibility to adjust their operating reactive power set-points, are defined either by grid-codes depicted in Fig. 2, when the case is denoted by $W P P(G C R)$, or by reactive power capability curves, illustrated in Fig. 4, when the case is denoted by WPP $(C C)$. In these two cases, only the reactive power set-points for WPPs are provided by the optimization routine. As mentioned earlier and depicted in Eq. (3), the control of WPPs' reactive power is performed indirectly by adjusting the voltages in their PoCs and therefore the control variables for the optimization routine in these cases only consist of WPPs' voltages:

$$
x=\left[V_{W P P_{1}}, V_{W P P_{2}}, V_{W P P_{3}}\right]^{T}
$$

TSO case - is defined as the case where the potential benefits of a coordination between TSO and DSO to minimize the losses in the distribution network are analyzed, by assuming that the OLTC of the $60 / 150 \mathrm{kV} \mathrm{TSO/DSO}$ transformer is controllable. Notice that this requires TSO to change the OLTC setting in accordance with the DSO requirements. The control vector in this case consists only of the voltage level of the OLTC transformer, controlled directly via tap-settings.

$$
x=\left[V_{T_{M V}}\right]^{T}
$$

It is worth noticing that the attention in the present investigations is mainly drawn to the impact that the control of the $60 / 150 \mathrm{kV}$ transformer has on the $60 \mathrm{kV}$ distribution network alone, the effects on the transmission side not being included 
TABLE II: Overview of simulated cases

\begin{tabular}{|c|c|c|c|}
\hline & \multicolumn{2}{|c|}{ WPP reactive power constraint } & OLTC \\
Case & GCR & CC & \\
\hline Base Case & - & - & - \\
\hline$W P P(G C R)$ & $\checkmark$ & - & - \\
\hline$W P P(C C)$ & - & $\checkmark$ & - \\
\hline$T S O$ & - & - & $\checkmark$ \\
\hline$T S O+W P P(C C)$ & - & $\checkmark$ & $\checkmark$ \\
\hline
\end{tabular}

in the network model, as out of the scope of the present study.

TSO + WPP $(\boldsymbol{C C})$ case - is defined as the case, where the reduction of active power losses by employing different available assets, such as for example, combining WPP reactive power control capabilities (CC) with the adjustment of the tap-changers of the TSO/DSO transformer, is investigated. In this case, both the reactive power-setpoints for WPPs and the OLTC tap-setting for the $60 / 150 \mathrm{kV}$ transformer are provided by the optimization process, while minimizing the losses. The control variables for the optimization routine are thus both the voltages at each individual WPP and the voltage at the OLTC transformer:

$$
x=\left[V_{W P P_{1}}, V_{W P P_{2}}, V_{W P P_{3}}, V_{T_{M V}}\right]^{T}
$$

\section{B. Active power losses}

High amount of wind power production in distribution networks leads to an increased reverse power flow into the system. In this respect, Fig. 5 shows how the active power exchange with TSO is impacted by the increased wind power production for the network under consideration. Notice that the power flow from TSO to DSO (TSO $\rightarrow$ DSO: + ) is completely reversed when the wind power production is more than $15 \mathrm{MW}$.

Furthermore, several studies [17], [34] in the past have shown, that reverse power flow in the network increases the power loss. This aspect, that high wind power generation in the network, as dominant contributor to reverse power flow, increases the active power loss, is also depicted in Fig. 6 . Notice that the region of WPP active power production larger than $15 \mathrm{MW}$ revealed in Fig. 5, accounts for only $40 \%$ of the total time simulated, but contributes with $89 \%$ to the total losses in the network. It should be mentioned that for the measurement data under consideration the TSO supplies reactive power to the DSO only for $31 \%$ of the total timestamps. Most of these timestamps are characterized by a reverse power flow in the network. Fig. 7 shows that $80 \%$ of the total active power losses in the network occur during these timestamps, or when TSO is supplying reactive power to the DSO . The results depicted in Fig. 5, 6, and 7 underline that it is efficient to utilize the local reactive power generation capabilities of WPPs to reduce network losses.

Table III provides an overview of energy losses (magnitude in $M W h$ and reduction in \%) for the network under consideration, as result output of the optimization routine for the different study cases. The energy losses for each case are

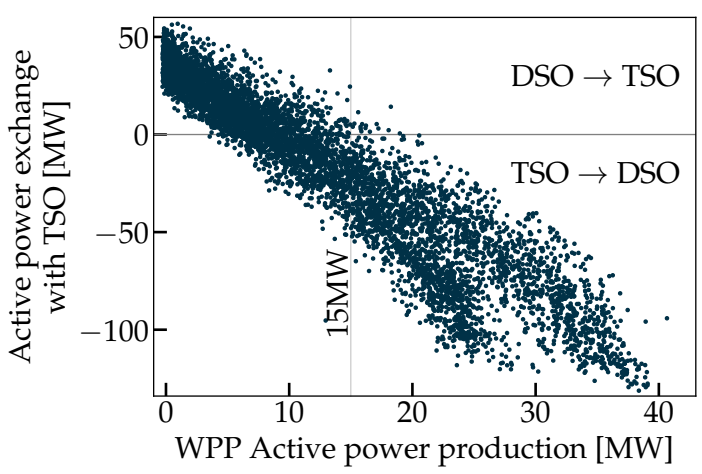

Fig. 5: WPP active power production Vs. active power flow from the transmission network

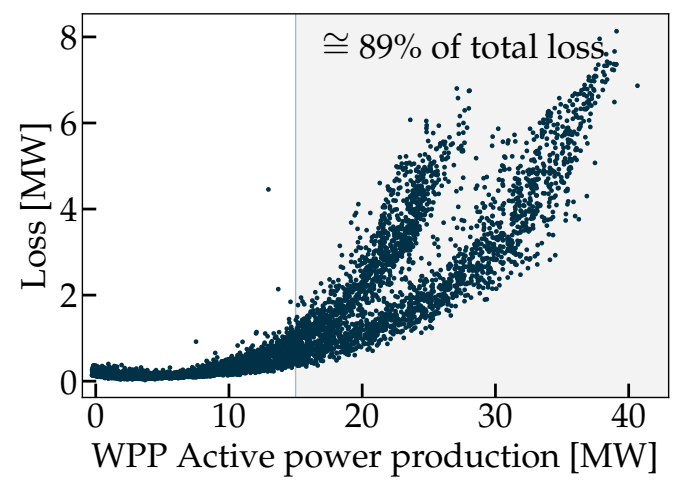

Fig. 6: WPP active power production Vs. distribution network loss

calculated by adding active power loss for all timestamps since the resolution of available data is 1 hour.

Based on WPPs' reactive power capabilities, which are considered in $W P P(G C R)$ and $W P P(C C)$ cases, the WPPs satisfy some of the reactive power demand in the network locally. Hence, the reactive power demand from the transmission network decreases and as a result, the current required to transfer the same amount of active power is also reduced, resulting in network loss reduction. This means that, an increase in

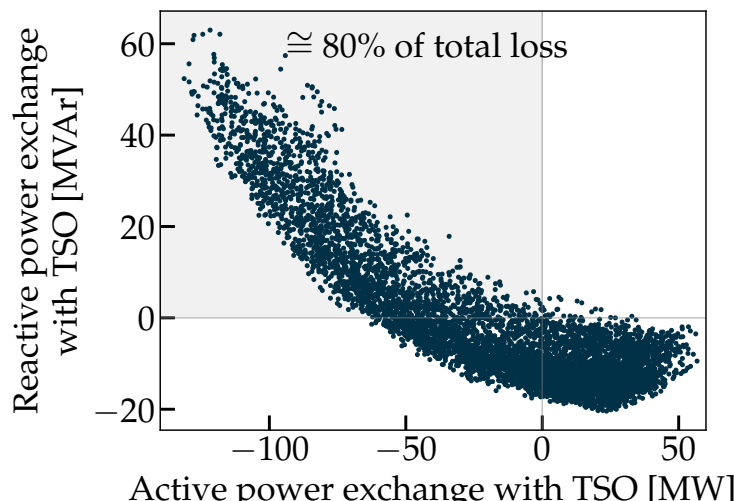

Fig. 7: Active power flow Vs. reactive power flow from TSO/DSO interface 
TABLE III: Energy losses - optimization results

\begin{tabular}{|c|c|c|}
\hline Case Name & Losses [MWh] & Reduction [\%] \\
\hline Base case & 8246.19 & - \\
\hline WPP (GCR) & 8030.459 & 2.6 \\
\hline WPP (CC) & 7899.329 & 4.2 \\
\hline TSO & 6739.511 & 18.2 \\
\hline TSO+WPP $(\mathbf{C C})$ & 6688.056 & $\mathbf{1 9}$ \\
\hline
\end{tabular}

the local supply of reactive power contributes to network loss reduction. This is justified by the simulation results over all the available data. Furthermore, as also depicted in Table III, the $W P P(C C)$ case has a greater loss reduction $(4.2 \%)$ than $W P P(G C R)$ case $(2.6 \%)$, due to the fact that, as mentioned before, the capability curves provides more room for reactive power support from WPPs.

As mentioned earlier, the current in the distribution network, which directly affects the network losses, can be decreased by changing the voltage profile in the network. The voltage level in the network can be altered by the tap-setting of TSO/DSO transformer and this is done in the TSO case. As shown in Table III, this results in a network loss reduction of $18.2 \%$. The final simulation case, $T S O+W P P(C C)$ augments the reduction of network losses due to increased voltage levels by enabling WPPs to supply reactive power locally. Simulation results for $T S O+W P P(C C)$ show a $19 \%$ loss reduction in the network. Notice that augmenting the TSO case with $W P P(C C)$ provides only a slight decrease in network losses ( $1 \%$ more), because higher node voltages limit the reactive power capabilities of WPPs, as depicted in Fig. 4.

\section{Voltage profile}

In all the simulated cases, voltages in the distribution network are expected to be higher than the base case for most of the time instances. For example, in $W P P(G C R)$ and $W P P(C C)$ cases, local reactive power support is the reason for voltage rise at the $60 \mathrm{kV}$ level, while in TSO case, the voltage profile is higher than the base case due to tap-changer settings. This is also vailable for $T S O+W P P(C C)$ case, as this is a combination of both the tap-changer settings and local reactive power from WPPs. As the voltage increases at the $60 \mathrm{kV}$ buses, the current required to transfer the same amount of power reduces proportionally. Active power loss in the network, which is proportional to the square of the current, thus reduces drastically. However, the node voltage not only depends on the reactive power support due to WPPs but also on the active power production and consumption in the network.

A distribution plot of the voltage at high voltage node of $W P P_{1}$ is plotted in Fig. 8 to provide an overall picture. The voltage distribution presented can be considered representative for all the other $60 \mathrm{kV}$ nodes in the network for the purpose of evaluating voltage profiles. This assumption is based upon the observation of voltages at all $60 \mathrm{kV}$ nodes for all timestamps. Note that the voltages at $10 \mathrm{kV}$ nodes or the low voltage nodes are not affected during any of the cases studied. Analysis of the voltage profiles is presented by grouping them into two parts.

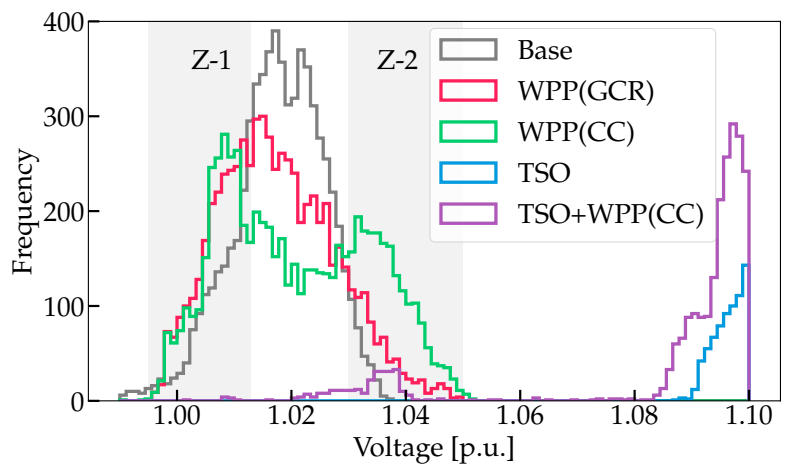

Fig. 8: Voltage profile distribution for $W P P_{1}$

$W P P(G C R)$ and $W P P(C C)$ : Two distinct zones of operations are observed for the $60 \mathrm{kV}$ node voltages in WPP only scenario, i.e. for $W P P(G C R) \quad W P P(C C)$, depicted by Z-1 and $\mathrm{Z}-2$ in Fig. 8. Fig. 8 suggests that, the $60 \mathrm{kV}$ node voltages in $W P P(C C)$ (green) case have a higher probability of being at voltage levels $\geq 1.03$ p.u. in comparison with $W P P(G C R)$ (red) or the base case (grey). Similarly, it can also be said that the voltages with $W P P(G C R)$ and $W P P(C C)$ have a higher probability of being $\leq 1.01$ p.u. as compared to the base case. The bi-modality in the two areas (Z-1 and Z-2) portrays two distinct region of operations, one where WPPs supply reactive power to the grid resulting in an increase in the node voltage, and the other being when WPPs consume reactive power from the grid resulting in a decrease in the node voltage.

TSO and TSO+WPP $(C C)$ : For the case when the OLTCs of the TSO/DSO transformer are actively controlled, the optimizer raises the overall $60 \mathrm{kV}$ voltage profile in the network close to the maximum voltage limits. As illustrated in Fig.4, the higher the voltage levels the lower reactive power capability of the WPPs according to $C C$ as illustrated in Fig.4. However, at some timestamps, the WPPs are still able to support the network with reactive power and contribute to further loss reduction. This is the reason for the small improvement in active power loss reduction from TSO to $T S O+W P P(C C)$ case.

\section{Reactive power}

The use of the reactive power contribution from the WPPs to reduce active power losses in the network is evaluated in the following for the 3 defined cases, where the reactive power from the WPPs is optimized, i.e. WPP $(G C R), W P P(C C)$ and $T S O+W P P(C C)$. Table IV provides an overview of WPPs' the reactive power contribution, as output of the optimization routine for the three considered cases. For the purpose of comparing WPP's reactive power contribution in each case, reactive power in case $W P P(G C R)$ is assumed $100 \%$, as WPPs do not contribute with reactive power in base case. Thus, the columns in Table IV denote percentage values of reactive power with respect to $W P P(G C R)$ case.

Notice that in $W P P(C C)$ case, WPPs reactive power consumption and supply increases by $7 \%$ and $113 \%$, respectively, compared to the $W P P(G C R)$ case. Furthermore, reactive power 
TABLE IV: WPPs' reactive power - optimization results $\%$ in reference to $W P P(G C R)$ case

\begin{tabular}{|c|c|c|}
\hline & \multicolumn{2}{|c|}{ Reactive power from WPPs [\%] } \\
Consume & Supply \\
\hline WPP (GCR) & 100 & 100 \\
\hline WPP (CC) & 107 & 213.1 \\
\hline TSO+WPP (CC) & 122.86 & 112.83 \\
\hline
\end{tabular}

consumption and supply of WPP increases by $22 \%$ and $12 \%$, respectively, in $T S O+W P P(C C)$ compared to the WPP $(G C R)$ case. It is worth underlying that, while comparing either $W P P(G C R)$ case or WPP $(C C))$ case with the $T S O+W P P(C C)$ case, other factors, such as the voltage profiles, also need careful consideration.

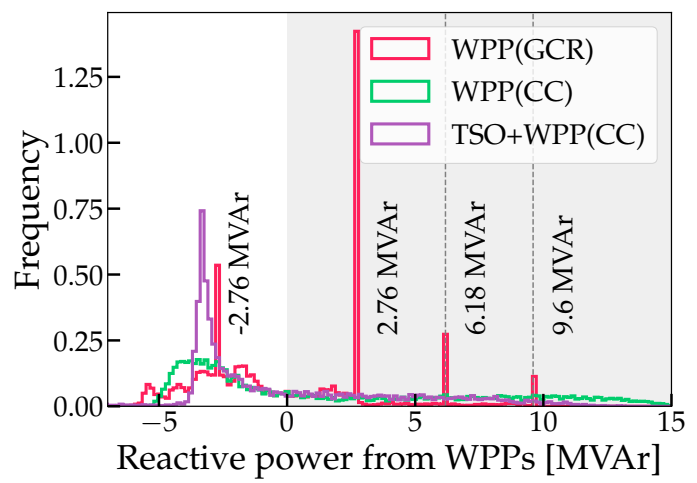

Fig. 9: Density distribution of reactive power from WPP

Fig. 9 depicts the density distribution of reactive power supplied by the WPPs in all three cases. The density distribution for $W P P(G C R)$ case shows 4 spikes at $-2.76 \mathrm{MVAr}$, 2.76MVAr, 6.18MVAr and 9.6MVAr which equates to the limit $2.28 \%$ of the installed capacities of the WPPs, which are $12 \mathrm{MW}, 15 \mathrm{MW}$ and $15 \mathrm{MW}$ as seen from Fig. 2. Since, GCR restricts WPP capabilities at the aforementioned values, spikes appear in the reactive power distribution of the WPPs in $W P P(G C R)$ case. According to the grid code [30], WPPs cannot contribute to reactive power at voltage higher or lower than $6 \%$ of the nominal voltage as noted from Fig. 2 . Notice that there are no such spikes in $W P P(C C)$ case. It is also noticeable that in $W P P(C C)$ and $T S O+W P P(C C)$ cases, WPPs are able to supply/absorb more reactive power than $W P P(G C R)$ case. Thus emphasizing the restrictiveness of $G C R$ in comparison with $C C$. It is worth noticing, that the reactive power capability of WPPs is limited in $T S O+W P P(C C)$ case due to high node voltages. This translates to the distribution of the reactive power being more concentrated around one value in $T S O+W P P(C C)$ case, whereas the distribution is more evenly distributed in $W P P(C C)$ case.

A comparison of reactive power from WPPs in $W P P(G C R)$ and $W P P(C C)$ case, along with the difference in active power loss reduction for the two cases is depicted and exemplified in Fig. 10 for $W \mathrm{PP}_{2}$. It also includes information on the hourly maximum reactive power limit for the WPPs. The fact that maximum reactive power capability of WPPs decreases with increased active power, as illustrated in Fig.4, is empirically depicted in Fig. 10 via the hourly upper limit of WPP reactive power. Thus, WPP active and reactive power are inverse proportional. Notice that for most of the active power range, the reactive power upper limit with $W P P(C C)$ is higher than the reactive power upper limit based on $W P P(G C R)$, which is $\pm 3.42 \mathrm{MVAr}$ for $W P P_{2}$. The most important observation from Fig. 10 is the scatter plot at the bottom, which portrays the difference between loss reduction in WPP(GCR) case and $W P P(C C)$ case. The term $\Delta$ Loss in Fig. 10 is given as follows,

$$
\Delta \text { Loss }=P_{L_{W P P(G C R)}}-P_{L_{W P P(C C)}}
$$

Thus, it can be concluded that, the higher reactive power contribution from WPPs in $W P P(C C)$ case translates to a higher active power loss reduction in comparison with $W P P(G C R)$ case.
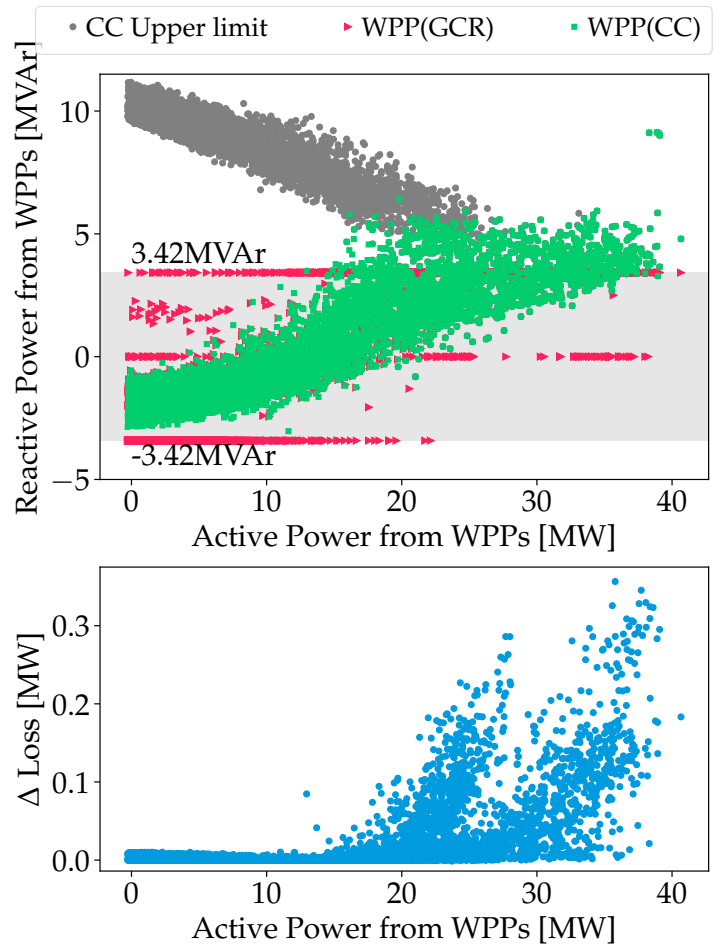

Fig. 10: Comparison of reactive power from $W P P_{2}$ in relation to active power and difference in the active power loss between $W P P(G C R)$ and $W P P(C C)$

Table $\mathrm{V}$ illustrates the reactive power exchange of the distribution network with the transmission network for the different cases. It is worth noticing that this exchange is also affected by the local supply and consumption of reactive power in the distribution network. For WPP(GCR) case, the distribution network imports (TSO $\rightarrow \mathrm{DSO}) \approx 22 \%$ less and exports $(\mathrm{DSO} \rightarrow \mathrm{TSO}) \approx 17 \%$ less reactive power to the transmission network as compared to the Base Case. The dependence of distribution network on the transmission network for reactive power further reduces with $C C$ where only $51 \%$ of reactive power is imported (TSO $\rightarrow \mathrm{DSO}$ ) in comparison to the base case. Note that in the TSO only case, the change in the reactive power transfer is due to the changing voltage profile in the network. In the TSO only case, the current in the distribution 
network reduces due to higher voltage levels. This makes the cables more capacitive. Hence, an increase in the reactive power being transferred from DSO to the TSO for the TSO only case is noticed.

The $95^{t h}$ percentiles indicated by dotted lines in Fig. 11 and tabulated in Table V suggest that with WPPs supplying reactive power locally, the demand of reactive power from the TSO decreases. Note that the $95^{t h}$ percentile values for both $W P P(G C R)$ and $W P P(C C)$ cases are smaller than $95^{\text {th }}$ percentile for base case. A similar result is observed in Fig. 12 where the $95^{\text {th }}$ percentile for $T S O+W P P(C C)$ case is at $21.276 \mathrm{MVar}$ as compared to $28.61 \mathrm{MVar}$ in TSO only case or a much higher value of 35.1 MVar in Base Case. The

TABLE V: Reactive power transfer between TSO-DSO: cumulative [\%] w.r.t. base case and percentiles optimization results

\begin{tabular}{|c|c|c|c|c|}
\hline \multirow{3}{*}{ Case Name } & \multicolumn{4}{|c|}{ Reactive power transfer } \\
\hline & \multicolumn{2}{|c|}{ TSO $\rightarrow$ DSO } & \multicolumn{2}{|c|}{ DSO $\rightarrow$ TSO } \\
\hline & $\sum_{[\%]}$ & $\begin{array}{c}95^{\text {th }} \text { percentile } \\
\text { [MVAr] }\end{array}$ & $\sum_{[\%]}$ & $\begin{array}{c}5^{\text {th }} \text { percentile } \\
\text { [MVAr] }\end{array}$ \\
\hline Base case & 100 & 35.1 & 100 & -16.65 \\
\hline WPP (GCR) & 78.13 & 29.3 & 83.1 & -13.6 \\
\hline WPP (CC) & 51 & 21.2 & 84.61 & -12.54 \\
\hline TSO & 74.2 & 28.6 & 122.69 & -18.8 \\
\hline TSO+WPP & 51.36 & 21.27 & 99.14 & -15.1 \\
\hline
\end{tabular}

density distribution of reactive power exchange between TSO and DSO gives a clear picture of how the reactive power requirements of the DSO shift in each scenario. Note that both Fig. 11 and Fig. 12, are negatively biased, i.e. the reactive power flows predominantly from the distribution network to the transmission network. It is important to note that, for $W P P(G C R)$ case and $W P P(C C)$ case, reactive power is imported from the transmission network only for $27 \%$ and $23 \%$ of the time, respectively. The data shows that, this exchange has more than $80 \%$ share in the network losses over the 10 simulated months. A similar phenomena is observed in TSO and $T S O+W P P(C C)$ cases, where the transmission network supplies reactive power to the distribution network for only $27 \%$ and $22 \%$ of the total time, and this exchange contributes $\approx 74 \%$ and $\approx 67 \%$ to the active power losses, respectively. Table V, Fig. 11, and Fig.12 suggests that the reactive power exchange between transmission and distribution network can be altered via WPP reactive power control. This reintroduces the distribution network as an asset to the transmission network for reactive power support.

\section{CONCLUSION}

An optimization methodology to minimize the losses in distribution networks with large share of wind power, exploiting wind power plants capability to control reactive power in coordination with the on-load tap changers (OLTCs) from the MV/HV transformer, is proposed and analysed. The study is conducted based on measurements from a real Danish distribution network data with a large share of controllable wind power plants (WPPs) under varying wind and load conditions. It is observed that the reactive power control capabilities of WPPs alone can contribute significantly to

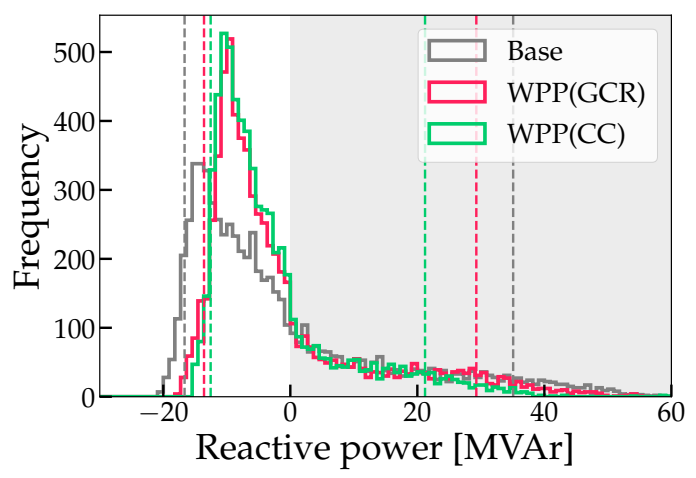

Fig. 11: Distribution of Reactive power transfer over TSO/DSO interface: $W P P(G C R)$ and $W P P(C C)$ case with $5^{t h}$ and $95^{t h}$ percentiles marked with dotted lines

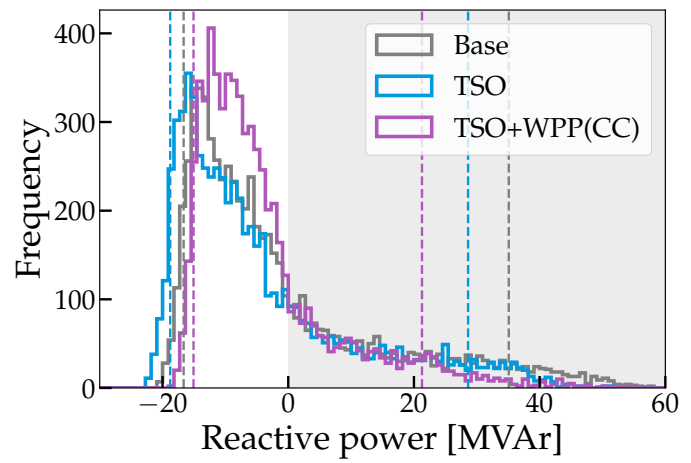

Fig. 12: Distribution of Reactive power transfer over TSO/DSO interface: TSO and TSO+WPP case with $5^{\text {th }}$ and $95^{\text {th }}$ percentiles marked with dotted lines

loss reduction in distribution networks. The reactive power control case was simulated using two different constraints, namely grid code requirements $(G C R)$ and capability curves $(C C)$. The results of the investigation show that the network losses decrease as the WPPs are able to provide more reactive power, whenever their operating reactive power set-points are defined based on capability curves. For example, in such situation, a loss reduction of $4.2 \%$ can be achieved for the considered network. Even better loss reductions $(\approx 19 \%)$ can be achieved by coordinating WPPs reactive power control capability and OLTC setting of the $60 / 150 \mathrm{kV}$ transformer. Upon analyzing the reactive power flow over the transmission system-distribution system (TSO/DSO) interface, it is found that, the reactive power exchange over the TSO/DSO interface can be altered via WPP reactive power control. The results clearly confirm that the distribution network with large share of wind power can support the transmission network by actively controlling the reactive power.

The results presented in this paper can be used for future studies to design an optimization methodology including weather dependent variability in the distribution network, and to greater possibilities of TSO/DSO coordination. 


\section{ACKNOWLEDGMENT}

This work has been carried out by the DTU Wind Energy Department during two different projects: NetVind (PSO project 2016 no. 12433) and WinGrid (Marie SklodowskaCurie grant agreement No 861398). Danish PSO-program ForskEL, administered by Energinet.DK, IEA Task-41, and the European Union's Horizon 2020 research and innovation program are acknowledged for funding this work. Norlys Forsyning $\mathrm{A} / \mathrm{S}$ is acknowledged for providing the network model and the measurement data for a real distribution network.

\section{REFERENCES}

[1] "Energistyrelsen — Data: Oversigt over energisektoren." [Online] Available: https://ens.dk/

[2] "Energinet." [Online]. Available: https://energinet.dk/

[3] F. Kalverkamp, B. Schowe-Von Der Brelie, T. D. Nguyen, T. Mertens, and M. Meuser, "Comparative analysis of European Grid Codes and compliance standards for distributed power generation plants with respect to future requirements of ENTSO-E and CENELEC," International ETG Congress 2015; Die Energiewende - Blueprints for the New Energy Age, pp. 605-610, 2015.

[4] K. Das, E. N. Martinez, M. Altin, A. D. Hansen, P. E. Sorensen, G. W. Thybo, M. Rangard, and K. M. Skytte, "Facing the challenges of distribution systems operation with high wind power penetration," 2017 IEEE Manchester PowerTech, Powertech 2017, 2017.

[5] K. L. Anaya and M. G. Pollitt, "Going smarter in the connection of distributed generation," Energy Policy, vol. 105, no. August 2016, pp. 608-617, 2017. [Online]. Available: http://dx.doi.org/10.1016/j.enpol.2017.01.036

[6] L. F. Ochoa and G. P. Harrison, "Minimizing energy losses: Optimal accommodation and smart operation of renewable distributed generation," IEEE Transactions on Power Systems, vol. 26, no. 1, pp. 198-205, 2011.

[7] Y. P. Agalgaonkar, B. C. Pal, and R. A. Jabr, "Distribution voltage control considering the impact of PV generation on tap changers and autonomous regulators," IEEE Transactions on Power Systems, vol. 29, no. 1, pp. 182-192, 2014.

[8] A. Allen and A. Allen, "Voltage Impacts of Utility-Scale Distributed Wind," no. September, 2014.

[9] S. C. Johnson, J. D. Rhodes, and M. E. Webber, "Understanding the impact of non-synchronous wind and solar generation on grid stability and identifying mitigation pathways," Applied Energy, vol. 262, no. December 2019, p. 114492, 2020. [Online]. Available: https://doi.org/10.1016/j.apenergy.2020.114492

[10] A. Baviskar, A. D. Hansen, K. Das, and M. Koivisto, "Challenges of Future Distribution Systems with a Large Share of Variable Renewable Energy Sources - Review," 19th Wind Integration Workshop 2020, no. November, 2020.

[11] J. H. Liu and C. C. Chu, "Long-Term Voltage Instability Detections of Multiple Fixed-Speed Induction Generators in Distribution Networks Using Synchrophasors," IEEE Transactions on Smart Grid, vol. 6, no. 4, pp. 2069-2079, 2015.

[12] M. Wilch, V. S. Pappala, S. N. Singh, and I. Erlich, "Reactive power generation by DFIG based wind farms with AC grid connection," 2007 IEEE Lausanne POWERTECH, Proceedings, pp. 626-632, 2007.

[13] S. Marin and A. Garces, "Robust optimal power flow in distribution systems with high penetration of wind energy using a model-based evolutionary strategy," 2014 IEEE PES Transmission and Distribution Conference and Exposition, PES T and D-LA 2014 - Conference Proceedings, vol. 2014-Octob, 2014.

[14] D. S. Stock, A. Venzke, L. Lower, K. Rohrig, and L. Hofmann, "Optimal reactive power management for transmission connected distribution grid with wind farms," IEEE PES Innovative Smart Grid Technologies Conference Europe, pp. 1076-1082, 2016.

[15] A. Soroudi, A. Rabiee, and A. Keane, "Distribution networks' energy losses versus hosting capacity of wind power in the presence of demand flexibility," Renewable Energy, vol. 102, pp. 316-325, 2017. [Online]. Available: http://dx.doi.org/10.1016/j.renene.2016.10.051

[16] A. Naeem, N. U. Hassan, and C. Yuen, "Power Loss Minimization in Power Distribution Systems Using Wind and Solar Complementarity," International Conference on Innovative Smart Grid Technologies, ISGT Asia 2018, pp. 1165-1170, 2018.
[17] F. R. Arduini, A. L. D. S. Pessoa, M. Oleskovicz, and E. N. Asada, "Analysis of the Integration of Type C Wind Turbines in Distribution Networks," 2019 IEEE Milan PowerTech, PowerTech 2019, 2019

[18] U. Jamil, N. Qayyum, A. Mahmood, and A. Amin, "Control Grid Strategies for Reduction of Real and Reactive Line Losses in Radial Power Distribution System," 1st International Conference on Electrical, Communication and Computer Engineering, ICECCE 2019, no. January 2020, pp. 1-5, 2019.

[19] L. Wenxue, L. Zhengtang, M. Changhui, Y. Dong, C. Bo, Z. Kang, Z. Ning, and L. Shan, "Reactive Power Optimization in Distribution Network Considering Reactive Power Regulation Capability and Fuzzy Characteristics of Distributed Generators," 2019 4th International Conference on Power and Renewable Energy, ICPRE 2019, pp. 387-393, 2019.

[20] S. H. E. Osman, G. K. Irungu, and D. K. Murage, "Impact of different locations of integrating SCIG wind turbine into distributed network using continuation power flow method," Proceedings of the International Conference on Computer, Control, Electrical, and Electronics Engineering 2019, ICCCEEE 2019, pp. 12-16, 2019.

[21] R. I. Cabadag, U. Schmidt, and P. Schegner, "Reactive power capability of a sub-transmission grid using real-time embedded particle swarm optimization," IEEE PES Innovative Smart Grid Technologies Conference Europe, vol. 2015-Janua, no. January, pp. 1-6, 2015.

[22] F. Yang and Z. Li, "Improve Distribution System Energy Efficiency with Coordinated Reactive Power Control," IEEE Transactions on Power Systems, vol. 31, no. 4, pp. 2518-2525, 2016.

[23] V. Calderaro, G. Conio, V. Galdi, G. Massa, and A. Piccolo, "Optimal decentralized voltage control for distribution systems with inverter-based distributed generators," IEEE Transactions on Power Systems, vol. 29 , no. 1, pp. 230-241, 2014.

[24] M. Tahir, R. A. El Shatshat, and M. M. Salama, "Reactive Power Dispatch of Inverter-Based Renewable Distributed Generation for Optimal Feeder Operation," 2018 IEEE Electrical Power and Energy Conference, EPEC 2018, pp. 1-6, 2018.

[25] L. Petersen, F. Iov, A. D. Hansen, and M. Altin, "Voltage Control Support and Coordination between Renewable Generation Plants in MV Distribution Systems," Tech. Rep., 0.

[26] A. Oulis-Rousis, D. Tzelepis, Y. Pipelzadeh, G. Strbac, C. D. Booth, and T. Green, "Provision of Voltage Ancillary Services through Enhanced TSO-DSO Interaction and Aggregated Distributed Energy Resources," IEEE Transactions on Sustainable Energy, vol. XX, no. X, pp. 1-1, 2020.

[27] Z. Tang, D. J. Hill, and T. Liu, "Distributed Coordinated Reactive Power Control for Voltage Regulation in Distribution Networks," IEEE Transactions on Smart Grid, vol. 12, no. 1, pp. 312-323, 2021.

[28] F.-p. n. 2016-1-12433, "Technical methodology for minimization of network losses," Tech. Rep. 2016, 2018.

[29] E.-E. N. Code, "Requirements for grid connection applicable to all generators," ENTSO-E: Brussels, Belgium, 2013.

[30] Energinet.dk, "Technical regulation 3.2.5 for wind power plants above $11 \mathrm{~kW}, "$ pp. 1-108, 2016.

[31] M. Sarkar, M. Altin, P. E. Sørensen, and A. D. Hansen, "Reactive power capability model of wind power plant using aggregated wind power collection system," Energies, vol. 12, no. 9, pp. 1-19, 2019.

[32] R. Nelson, "Full-converter Wind Turbine Technology," 2014 [Online]. Available: http://home.eng.iastate.edu/ jdm/wesep594/Fullconverter WTG Technology.pdf

[33] D. Nedic, "Tap Adjustment in AC Load Flow," 2002.

[34] M. Delfanti, D. Falabretti, and M. Merlo, "Dispersed generation impact on distribution network losses," Electric Power Systems Research, vol. 97, pp. 10-18, 2013. 